\title{
Delta and Theta waves in Super Consciousness Meditation.
}

Dr Bheemaiah, Anil Kumar, Mutter Gottlich, Seattle WA 98125.

miyawaki@yopmail.com

\section{Abstract.}

Study on Kundalini Meditation of Super-conscious Meditation of the Himalayan Tradition and Sahaja Meditation, to determine the average power spectral densities and power ratios of TP9, AF7, AF8, and TP10, electrodes and two ear electrodes on a Muse Headset.

These parameters are used to create quantitative criteria to indicate degree of meditation and to create a trigger for bird chirp events.

We find an increase in Delta and Theta wave power densities, in the deep meditation state as compared to the initiation and restful states.

keywords: Kundalini, super consciousness, neurosky, muse, chakra based meditation, alpha to beta ratio, delta to beta ratio, power spectral densities, differential power spectral densities, fMRi, time series, iD convolutional networks. lyapunov coefficient

\section{Introduction.}

The chakra based system is the basis of kundalini meditation, popularized in the west, in Sahaja meditation, by late Mata Nirmalaji and as Super Consciousness meditation by late Swami Rama, of Himalayan Institute. There is a spectrum of literature on the topic of EEG signatures of kundalini based meditation with respect to fMRI and power spectral differential and averages from EEG data.

A higher beta wave ratio to alpha and delta is reported in many studies, while an increased delta and theta compared to alpha and beta waves is the general consensus of the EEG meditation community.

In this paper we use wearable based EEG gear with seven or less electrodes and develop heuristics for the evaluation of the meditation bardos. 


\section{Materials and methods}

1. Records with muse headsets.

We recorded with muse I headsets.Muse (RRID:SCR_014418)

\subsection{Original contributions.}

We demonstrate using the Muse headset and the Muse EEG record software that there is a transition from alpha to beta to delta to theta wave as one meditated in Kundalini meditation progresses to the Sahasrara Chakra. Contrary to much published research that reports an increase in beta waves by 60 to 120 percent [refs], We observed a greater than $120 \%$ increase in delta waves and a 20 to 50 percent increase in theta waves in deep meditation Waves-form in resting stages.

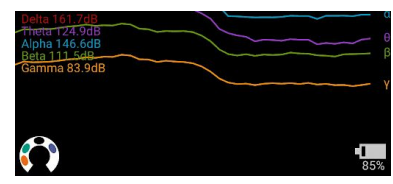

\subsection{Discussion.}

Sleep Bardos, are comparable in the EEG signature to the fourth state of consciousness, as described in the creative intelligence(Jedrczak, Beresford, and Clements 1985) formulation of T.M, as delta cycles, similar to published literature on delta wave induced sleep. (Bheemaiah, n.d.).

While many newer eeg based wearables are available in the market, neurosky, has developed an asic, amenable to research, by integration in wearables.(Pantech 2018). The paper presents research findings towards algorithms for interfacing with several such wearable EEG based headsets. In this paper, the author used the MUSE I headset.

("[No Title]" n.d.) describes, kundalini meditation, the use of yantras and mandalas in meditation and the various chakras in the formulation of a theoretical basis for the kundalini system as super consciousness.

\subsection{Future work.}

A clinical study on a significant population of Kundalini meditators at Hiht Dehradun, India will supplement this publication. A theoretical analysis of the EEG results is being carried out using a variety of mathematical transformations, including a Fourier transformation and analysis by normalization, a generalized transformation study and chaotic analysis. We also used a 1D $\mathrm{R}-\mathrm{CNN}$ to learn meditation niche patterns for individualized feedback on the super-conscious meditation. We give the therapeutic effect of the meditation, this supplements the present software available through interaxon Inc, indicating the progression of the meditation through each chakra and the use of the meditation for improved concentrations and for the treatment of 
psychosis spectrum disorder and depression and bipolar. We also needed to formulate the criteria for bird chirp and consciousness level indications for Kundalini meditation.

\subsection{Methods.}

meditation was performed with the Muse headset and a specialized Android Application Log EEG waves, performed at Maharishi University of Management, Center for Brain, consciousness and Cognition. Subjects: Dr Bheemaiah, Anil Kumar. Measurements are from TP9, AF7, AF8, and TP10, electrodes and two ear electrodes. The average power level and power density are plotted with time for an algorithmic measure of absolute power spectrum level and beta/alpha and delta/theta ratio. These ratios indicate levels of meditation by a quantitative index and a trigger for a bird chirp. MUSE (RRID: SCR_014418) It is a portable scalp electroencephalography (EEG) system that is used to measure brain activity. It is battery powered and has four active electrodes located at 10-20 coordinated TP9, AF7, AF8, and TP10, a common mode referenced, and a driven right leg. It includes an accelerometer and gyroscope, and works with desktop and mobile EEG acquisition and visualization software.

\section{Results}

1. Records with muse headsets.

\section{Plot of Delta and Beta Waves during Deep Meditation}

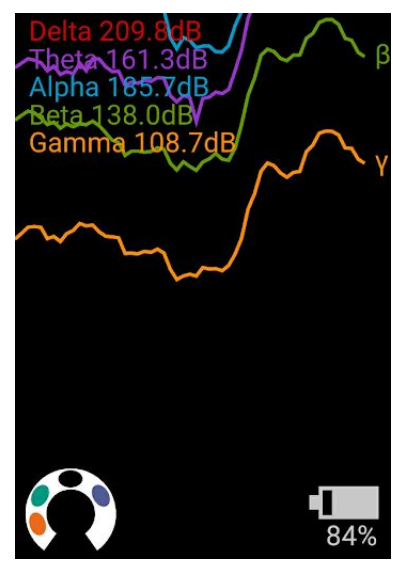


Plot Of Delta and Beta Waves in Initiation Stage.

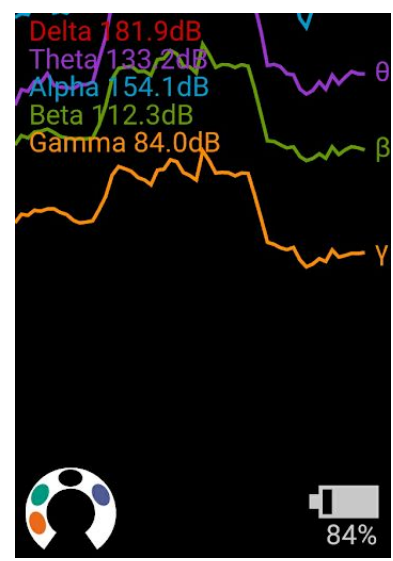

\section{References}

Muse Research: DOI:10.1371/journal.pone.0130129

Bheemaiah, Anil Kumar. n.d. "Reversible and Non Lethal Weapons: A Path to Disarmament." https://doi.org/10.31219/osf.io/6squ3.

Jedrczak, Andrew, Michael Beresford, and Geoffrey Clements. 1985. "The TM-Sidhi Program, Pure Consciousness, Creativity and Intelligence." The Journal of Creative Behavior. https://doi.org/10.1002/j.2162-6057.1985.tb00409.x.

"[No Title]." n.d. Accessed June 5, 2020. https://vixra.org/pdf/1909.0153v1.pdf.

Pantech. 2018. "Low Cost EEG Headset in India - Pantech Blog." Pantech Blog. November 13, 2018. https://www.pantechsolutions.net/blog/low-cost-eeg-headset-in-india/. 